The Annals of Statistics

2002, Vol. 30, No. 5, 1496-1511

\title{
CLEAR TWO-FACTOR INTERACTIONS AND MINIMUM ABERRATION
}

\author{
By HUAIQING WU ${ }^{1}$ AND C. F. J. WU ${ }^{2}$ \\ Iowa State University and University of Michigan
}

\begin{abstract}
Wu and Hamada recommend selecting resolution IV designs with the maximum number of clear two-factor interactions (2FIs), called MaxC2 designs. In this paper, we develop a method by using graphical representations, combinatorial and group-theoretic arguments to prove if a given design is a MaxC2 design. In particular, we show that all known minimum aberration designs with resolution IV are MaxC2 designs (except in six cases) and that the second $2^{9-4}, 2^{13-7}, 2^{16-10}$ and $2^{17-11}$ designs given in $\mathrm{Wu}$ and Hamada are MaxC2 designs. The method also enables us to identify new MaxC2 designs that are too large to be verified by computer search.
\end{abstract}

1. Introduction. The minimum aberration (MA) criterion is commonly used for selecting optimal $2^{k-p}$ fractional factorial designs. In some situations, however, other criteria can lead to better designs. A two-factor interaction (2FI) is called clear if it is not aliased with any main effects or other 2FIs. This is explained in $\mathrm{Wu}$ and Hamada [(2000), Chapter 4]. They proposed a general rule for selecting $2^{k-p}$ designs with maximum resolution IV. That is, among resolution IV designs with given $k$ and $p$, those with the maximum number of clear 2FIs (MaxC2) are the best. Such designs will be called MaxC2 designs. Note that MA designs are not necessarily good according to this criterion. For example, the MA $2^{15-9}$ design given in $\mathrm{Wu}$ and Hamada [(2000), page 197] contains no clear 2FIs, but another $2^{15-9}$ design of resolution IV given there has 27 clear 2FIs. In this paper, an approach is developed to prove if a given design is a MaxC2 design. The main tools (given in Sections 2 and 4) are a graph representation and classification of length- 4 words and some useful identities and bounds on the number of clear 2FIs. These tools can reduce the search for designs to a much smaller set. Together with other combinatorial and group-theoretic arguments, they are effective in proving known and new designs to be MaxC2 designs. A summary of techniques in the proposed approach and concluding remarks are given in Section 5.

A regular $2^{k-p}$ fractional factorial design is determined by its defining contrast subgroup, which consists of $2^{p}-1$ defining words. The vector $W=\left(A_{1}, \ldots, A_{k}\right)$ is called the word-length pattern, where $A_{i}$ denotes the number of words of length $i$ in the defining contrast subgroup. The resolution of a design is defined as the

Received November 2000; revised June 2001.

${ }^{1}$ Supported in part by Iowa State University Research Grant.

${ }^{2}$ Supported in part by NSF Grant DMS-00-72489 and NSA Grant MSPR-00G-091. AMS 2000 subject classification. $62 \mathrm{~K} 15$.

Key words and phrases. Alias set, defining contrast subgroup, resolution, word-length pattern. 
smallest $j$ with $A_{j} \geq 1$. To discriminate designs of the same resolution, Fries and Hunter (1980) proposed the following criterion. For two designs $d_{1}$ and $d_{2}$ with $s$ being the smallest value such that $A_{s}\left(d_{1}\right) \neq A_{s}\left(d_{2}\right), d_{1}$ is said to have less aberration than $d_{2}$ if $A_{s}\left(d_{1}\right)<A_{s}\left(d_{2}\right)$. If there is no design with less aberration than $d_{1}$, then $d_{1}$ has minimum aberration (MA).

It is well known that there exist no designs with resolution at least IV if $k>2^{k-p-1}$ [see Bose (1947)]. For $2^{k-p-2}+1<k \leq 2^{k-p-1}$, the maximum resolution of a $2^{k-p}$ design is IV, but in this case, Chen and Hedayat (1998) showed that no resolution IV designs can have any clear 2FIs. Let $k_{\max }(q)$ denote the maximum value of $k$ for which there exists a $2^{k-(k-q)}$ design with resolution at least $\mathrm{V}$. Then for $k_{\max }(k-p)<k \leq 2^{k-p-2}+1$, there exist no designs with resolution at least $\mathrm{V}$, but there exist resolution IV designs with clear 2FIs [see Chen and Hedayat (1998)]. This is the case to which the previous rule of $\mathrm{Wu}$ and Hamada should apply. Throughout this paper, we will focus on this case. As we will see later, MA designs can be MaxC2 designs in this case, especially when the number of length- 4 words is small. However, when $k$ is close to $2^{k-p-2}+1$, MA designs may have no clear 2FIs while MaxC2 designs can have a large number of clear 2FIs.

For $k_{\max }(k-p)<k \leq 2^{k-p-2}+1$, MA designs for 32 and 64 runs and for 128 runs with $12 \leq k \leq 14$ have been obtained, either through theoretical derivations or via computer search; see the tables in Wu and Hamada [(2000), Chapter 4]. In Section 3, we use the proposed method to show that, these MA designs are also MaxC2 designs, except for $2^{9-4}, 2^{13-7}, 2^{14-8}, 2^{15-9}, 2^{16-10}$ and $2^{17-11}$ designs. We also show in Section 4 that the second $2^{9-4}, 2^{13-7}, 2^{16-10}$ and $2^{17-11}$ designs given in Wu and Hamada [(2000), pages 195 and 197] are MaxC2 designs. These designs are obtained by Chen, Sun and Wu (1993) via computer search. The cases of $2^{14-8}$ and $2^{15-9}$ designs are still under investigation. The method also enables us to prove that a class of designs due to Tang, Ma, Ingram and Wang (2002) are MaxC2 designs, which cannot be done by computer search.

As the work of Chen (1998) indicated, even for 128-run designs with $k=13$ and 14, finding MA designs via computer search is not an easy task. For 128-run designs with $15 \leq k \leq 33$, little work has been done for finding either MA or MaxC2 designs. This is in part because there is no complete catalog of 128-run designs. Since in these cases, MaxC2 designs are the best according to the $\mathrm{Wu}-$ Hamada rule, it is of particular interest to find designs that have many clear 2FIs. The results in this paper reveal some intrinsic structures of the MaxC2 designs that can potentially be used to construct designs with many clear 2FIs for large run size and to provide useful information for finding MaxC2 designs via computer search. For example, we are able to find a new MaxC2 $2^{15-8}$ design by employing the proposed method to reduce the search to a very small set of designs and use computer enumeration to finish the job. 
2. A graphical representation of length-4 words. Let $d$ be a $2_{\mathrm{IV}}^{k-p}$ design, where the subscript IV indicates that $d$ is of resolution IV. Let $A_{4}(d)$ be the number of its length-4 words, $L_{4}$ the set of its length-4 words, $C(d)$ the number of clear 2FIs and

$$
U(d)=k(k-1) / 2-C(d)
$$

the number of unclear 2FIs. Consider a graph consisting of vertices and lines, where each vertex represents an independent word in $L_{4}$, and a line connects two vertices if their product is a length- 4 word. If the product of three or four vertices is a length-4 word, they are indicated by unfilled circles or boxes, respectively. Such a graph can be used to show relationships among some or all length-4 words.

Let $g_{(}\left(w_{1}, \ldots, w_{m}\right)$ denote the set generated by $m$ independent length-4 words $w_{1}, \ldots, w_{m}$. Here, "generated" means taking products of $w_{1}, \ldots, w_{m}$ of various lengths. For example, $g\left(w_{1}, w_{2}, w_{3}\right)=\left\{w_{1}, w_{2}, w_{3}, w_{1} w_{2}, w_{1} w_{3}, w_{2} w_{3}\right.$, $\left.w_{1} w_{2} w_{3}\right\}$. Then

$$
G_{m}=G\left(w_{1}, \ldots, w_{m}\right)=L_{4} \cap g\left(w_{1}, \ldots, w_{m}\right)
$$

is called the subset of $L_{4}$ generated by $m$ independent length- 4 words $w_{1}, \ldots, w_{m}$. A graph is called the graph of $d$ if it represents $L_{4}$, and a subgraph of $d$ if it represents $G_{m}$ for some independent length-4 words $w_{1}, \ldots, w_{m}$. Figures 1 (a)-(h) and 2(a)-(n) give all possible subgraphs of $d$ (i.e., graphs for $G_{m}$ ) for $m \leq 4$. Detailed constructions of these graphs are given below. Let $l$ be the number of length-4 words in $G_{m}$. Clearly, $m \leq l \leq 2^{m}-1$. Throughout this section, all length-4 words under consideration are in $G_{m}$.

It is obvious that the subgraph of $d$ for $m=1$ is Figure 1(a). (The only length-4 word $w_{1}$ is represented by a vertex.) For $m=2, l=2$ or 3 . If $l=2, w_{1}$ and $w_{2}$ do not share two common letters, and are represented by two separate vertices in Figure 1(b). For $l=3, w_{1} w_{2}$ is a length-4 word, and any two of $w_{1}, w_{2}$ and $w_{1} w_{2}$ share two common letters and their product gives the third length- 4 word.

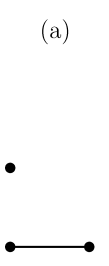

(e)

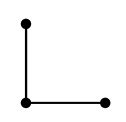

(f) (b)

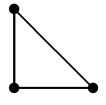

(g)

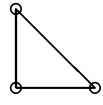

(h)

FIG. 1. Graphs of length-4 words: (a) $m=l=1$, (b) $m=l=2$, (c) $m=2, l=3$, (d) $m=l=3$, (e) $m=3, l=4$, (f) $m=3, l=5$, (g) $m=3, l=6$, and (h) $m=3, l=7$. 
We call these three words a tri-word group. They can be written as $A B C D, A B E F$ and $C D E F$, and represented by two vertices connected by a line, as shown in Figure 1(c). For $m=3$ and 4, the following lemma will be used.

LeMmA 2.1. (i) Suppose that $v_{1}, v_{2}, v_{3}, u=v_{1} v_{2} v_{3} \in G_{m}$. Then at least one of $v_{1} v_{2}, v_{1} v_{3}$ and $v_{2} v_{3}$ is a length-4 word. Thus, $u$ is the product of two length-4 words $u_{1}$ and $u u_{1}$ in $G_{m}$, where $u_{1}$ is one of $v_{1}, v_{2}$ and $v_{3}$.

(ii) If $m=4, l=5$ and $v=w_{1} w_{2} w_{3} w_{4} \in G_{4}$, any two of $w_{1}, \ldots, w_{4}$ share $a$ common letter and any three of them do not.

(iii) If $m=4$ and $l \geq 6$, there exist $l-4$ different tri-word groups.

(iv) Any two different tri-word groups share at most one common word.

PROOF. (i) Let $a_{i}$ be the number of letters that occur $i$ times in $v_{1}, v_{2}$ and $v_{3}$. Since $v_{1}, v_{2}$ and $v_{3}$ are length- 4 words, $a_{1}+2 a_{2}+3 a_{3}=12$. Since $u=v_{1} v_{2} v_{3}$ is a length- 4 word, $a_{1}+a_{3}=4$. Thus, $a_{2}+a_{3}=\left\{\left(a_{1}+2 a_{2}+3 a_{3}\right)-\left(a_{1}+a_{3}\right)\right\} / 2=$ $(12-4) / 2=4$; that is, four letters occur at least twice in $v_{1}, v_{2}$ and $v_{3}$. This implies that at least one of the three pairs $\left(v_{1}, v_{2}\right),\left(v_{1}, v_{3}\right)$ and $\left(v_{2}, v_{3}\right)$ shares two common letters, say $v_{1}=A B C D$ and $v_{2}=A B E F$. Then $v_{1} v_{2}$ is a length- 4 word and $u=\left(v_{1} v_{2}\right) v_{3}$ is the product of two length-4 words $v_{3}$ and $u v_{3}=v_{1} v_{2}$ in $G_{m}$.

(ii) Let $a_{i}$ be the number of letters that occur $i$ times in $w_{1}, \ldots, w_{4}$. Since $w_{1}, \ldots, w_{4}$ are length-4 words, $a_{1}+2 a_{2}+3 a_{3}+4 a_{4}=16$. Since $v=w_{1} w_{2} w_{3} w_{4}$ is a length- 4 word, $a_{1}+a_{3}=4$. Thus,

$$
\begin{aligned}
a_{2}+a_{3}+2 a_{4} & =\left\{\left(a_{1}+2 a_{2}+3 a_{3}+4 a_{4}\right)-\left(a_{1}+a_{3}\right)\right\} / 2 \\
& =(16-4) / 2=6 .
\end{aligned}
$$

Since $l=5$ and $v$ is a length- 4 word, none of the six pairs $\left(w_{i}, w_{j}\right)(1 \leq i<j \leq 4)$ share two common letters. Because no pair can share three common letters, any pair can share only one or no letter in common. This implies $a_{4}=0$ or 1 . If $a_{4}=1$, $a_{2}=a_{3}=0$, which contradicts (2.3). If $a_{4}=0$ and three of $w_{1}, \ldots, w_{4}$ (say $w_{1}, w_{2}$ and $w_{3}$ ) share a common letter, $a_{3}=1$ and $w_{1}, w_{2}$ and $w_{3}$ can be represented by $A B C D, A E F G$ and $A H J K$, respectively. Then $w_{1} w_{2} w_{3}$ is of length 10 , and hence no matter how $w_{4} \in G_{4}$ is chosen, $w_{1} w_{2} w_{3} w_{4} \in G_{4}$ cannot be satisfied. Thus $a_{4}=0$ and $a_{3}=0$, which implies $a_{2}=6$ via (2.3), completing the proof.

(iii) Let $S_{1}=\left\{w_{1}, \ldots, w_{4}\right\}, S=G_{4} \backslash S_{1}, S_{2}=S \cap\left\{w_{i} w_{j}: 1 \leq i<j \leq 4\right\}$ and $S_{3}=S \cap\left\{w_{i} w_{j} w_{k}: 1 \leq i<j<k \leq 4\right\}$. For $w_{i} w_{j} \in S_{2}$, there is a tri-word group $\left\{w_{i}, w_{j}, w_{i} w_{j}\right\}$. For $u \in S_{3}$, by part (i), there exists $u_{1} \in S_{1}$ such that $u u_{1} \in S_{2}$. This gives a tri-word group $\left\{u_{1}, u u_{1}, u\right\}$. We show that these tri-word groups are different from each other. Otherwise, suppose two of them, obtained from $u_{2}, u_{3} \in S_{2} \cup S_{3}$, equal $T_{0}$. Then $u_{2}, u_{3} \in T_{0}$, implying that $T_{0}=\left\{u_{2}, u_{3}, u_{2} u_{3}\right\}$. For any $w \in S_{2}$, its tri-word group $T_{1}$ contains two words in $S_{1}$. Thus $T_{1} \neq T_{0}$, implying that $u_{2}, u_{3} \notin S_{2}$. Then $u_{2}, u_{3} \in T_{0} \cap S_{3}$. This is a contradiction since any of the above tri-word groups contains at most one word in $S_{3}$. 
If $v=w_{1} w_{2} w_{3} w_{4} \in G_{4}$, since $l \geq 6$, there exists a length-4 word $w \in S_{2} \cup S_{3}$. If $w \in S_{2}$, say, $w=w_{1} w_{2}, v$ is the product of three length-4 words $w, w_{3}$ and $w_{4}$. By part (i), $v$ is also the product of two length-4 words $v_{0}$ and $v v_{0}$. If $w \in S_{3}$, $v_{0}=w v \in S_{1}$ and $v v_{0}=w \in S_{3}$. Again $v$ is the product of two length-4 words $v_{0}$ and $v v_{0}$. Thus there is a tri-word group $\left\{v_{0}, v v_{0}, v\right\}$, which is different from the above tri-word groups obtained from words in $S_{2} \cup S_{3}$, since none of them contain $v$. In summary, we have obtained $l-4$ different tri-word groups, one for each length-4 word in $S$.

(iv) If two tri-word groups share two common words $u_{1}$ and $u_{2}$, they must equal $\left\{u_{1}, u_{2}, u_{1} u_{2}\right\}$, contradicting the assumption that they are different.

Proposition 2.1. For any $2_{\mathrm{IV}}^{k-p}$ design $d$, all the possible subgraphs of $d$ for $m=3$ and 4 are given by Figures $1(\mathrm{~d})-(\mathrm{h})$ and 2(a)-(n), respectively.

REMARK. The condition $m \leq 4$ is not as restrictive as it may appear because these results can apply to designs whose graphs contain more than four independent length-4 words. Working on the subgraphs of these designs for $m=4$ can often reduce the search for MaxC2 designs to a much smaller set; see, for example, the MaxC2 $2^{12-6}$ and $2^{15-8}$ designs given in Sections 3 and 4.

PROOF OF PROPOSITION 2.1. For $m=3$, if none of $w_{1} w_{2}, w_{1} w_{3}$ and $w_{2} w_{3}$ are length-4 words, it follows from Lemma 2.1(i) that $w_{1} w_{2} w_{3}$ is not a length-4 word. Thus $l=3$ and $w_{1}, w_{2}$ and $w_{3}$ are represented by three separate vertices, as in Figure 1(d). If at least one of $w_{1} w_{2}, w_{1} w_{3}$ and $w_{2} w_{3}$ is a length-4 word, say $w_{1} w_{2} \in G_{3}, w_{1}$ and $w_{2}$ share two common letters and can be written as $w_{1}=A B C D$ and $w_{2}=A B E F$. Thus $w_{1} w_{2}=C D E F$. Suppose $w_{3}$ contains $t$ letters from $A, \ldots, F$. For $t=0$ or $1, w_{3}$ does not share two common letters with any of $w_{1}, w_{2}$ or $w_{1} w_{2}$, as represented by Figure $1(\mathrm{e})$, with vertices $w_{1}, w_{2}$ and $w_{3}$. For $t=2$, since the pairs $A B, C D$ and $E F$ are symmetric and two letters within each pair are symmetric, it suffices to consider $w_{3}=A B G H$ or $A C G H$. If $w_{3}=A C G H$, we have Figure 1(f), with vertices $A B C D, A B E F$ and $A C G H$. If $w_{3}=A B G H$, we have Figure $1(\mathrm{~g})$, with vertices $A B C D, A B E F$ and $A B G H$, which generate six length-4 words, called a six-word loop. For $t \geq 3$, any of $A B, C D$ or $E F$ cannot occur in $w_{3}$. Otherwise, say $A B$ and $C$ occur in $w_{3}$, then $w_{3} A B C D$ would be a length-2 word, contradicting the assumption that $d$ is of resolution IV. Thus it suffices to consider $w_{3}=A C E G$, since two letters within each pair $A B, C D$ or $E F$ are symmetric. Then we have Figure 1(h), with vertices $A B C D, A B E F$ and $A C E G$, which generate seven length-4 words, called a seven-word group. Note that the vertices are unfilled circles, indicating that the product of three vertices is a length-4 word.

For $m=4$, many more cases need to be considered. Clearly, for $l=4$, we have Figure 2(a). For $l=5$, if one of $w_{i} w_{j}(1 \leq i<j \leq 4)$ is a length-4 word, we have Figure 2(c), with vertices $w_{1}, \ldots, w_{4}$. If none of $w_{i} w_{j}(1 \leq i<j \leq 4)$ are 


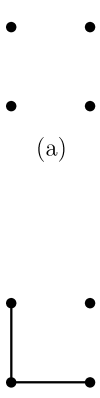

(e)

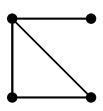

(i)

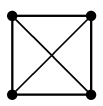

$(\mathrm{m})$

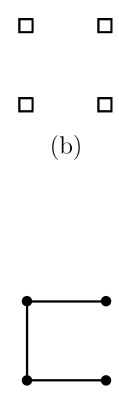

(f)

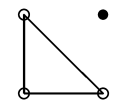

(j)

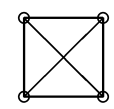

(n)

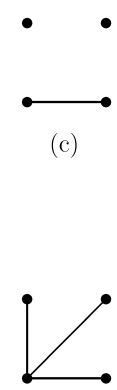

(g)

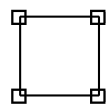

(k)

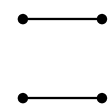

$(\mathrm{d})$

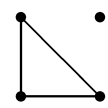

(h)

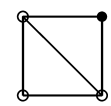

(1)

FIG. 2. Graphs of length-4 words for $m=4$ : (a) $l=4$, (b) $l=5$ (without a line), (c) $l=5$ (with a line), (d), (e) $l=6$, (f), (g) $l=7$ (without a six-word loop), (h) $l=7$ (with a six-word loop), (i) $l=8$ (with a six-word loop), (j) $l=8$ (with a seven-word group), (k) $l=9,(1),(\mathrm{m}) l=10,(\mathrm{n}) l=14$.

length-4 words, it follows from Lemma 2.1(i) that none of $w_{i} w_{j} w_{k}(1 \leq i<j<$ $k \leq 4)$ are length-4 words. Hence $w_{1} w_{2} w_{3} w_{4} \in G_{4}$ and we have Figure 2(b). By Lemma 2.1(ii), the four vertices can be written as $A B C D, A E F G, B E H J$ and $C F H K$. Since the product of the four vertices is a length-4 word, they are represented by unfilled boxes.

For $l=6$, by Lemma 2.1(iii) and (iv), there exist two different tri-word groups with at most one common word. If they share no common word, they must contain four independent length-4 words and we have Figure 2(d). (If they contain only three independent length- 4 words, $m=3$ and one of the graphs in Figure 1 would contain two disjoint tri-word groups, which is not the case.) Otherwise, their common word is represented by a vertex with two lines connected to it and another independent length- 4 word not in the groups is represented by a separate vertex, as in Figure 2(e). For $l=7$, by Lemma 2.1(iii) and (iv), there exist three different triword groups with at most one common word between any two of them. If the three groups share one common word, it can be represented by a vertex with three lines connected to it, as in Figure 2(g). If two of the three groups do not share a common word, they contain six length- 4 words. Since $l=7$, the third group contains only 
one word not in the two groups and thus has a common word with each of them. This can be represented by Figure 2(f). If any two groups share a common word not in the third group, the three groups contain a six-word loop, which is generated by the three common words. Thus there is another independent length- 4 word. This can be represented by Figure 2(h). It remains to consider $m=4$ and $l \geq 8$.

Suppose first that $G_{4}$ does not contain any six-word loop or seven-word group. Then Figure 1(f) is a subgraph of $d$ and its three vertices can be written as $w_{1}=A B C D, w_{2}=A B E F$ and $w_{3}=A C G H$. Hence $w_{1} w_{2}=C D E F$, $w_{1} w_{3}=B D G H, w_{2} w_{3}=B C E F G H$ and $w_{1} w_{2} w_{3}=A D E F G H$. Suppose another independent length-4 word $w_{4}$ contains $t$ letters from $A, \ldots, H$. Since $l \geq 8$, $t \geq 2$. [Otherwise, the graph for $G_{4}$ would be Figure $2(\mathrm{e})$ and then $l=6$.] If $t=2, w_{2} w_{3} w_{4}$ and $w_{1} w_{2} w_{3} w_{4}$ cannot be length-4 words. Since $l \geq 8$, there are two more length- 4 words of the form $w_{4} u$ and $w_{4} v$, where $u$ and $v$ belong to $\left\{w_{1}, w_{2}, w_{3}, w_{1} w_{2}, w_{1} w_{3}\right\}$. Denote the two letters in $w_{4}$ that are from $A, \ldots, H$ by $x$ and $y$. Then both $u$ and $v$ contain $x$ and $y$. Since $u$ and $v$ cannot share three letters in common, they must share two letters in common. Then $w_{4}, u$ and $v$ would lead to a six-word loop, contradicting the assumption that $G_{4}$ does not contain any six-word loop. Thus $t \geq 3$. If $w_{4}$ contains $E F$ (or $G H$ ) for which $d$ is of resolution IV, $w_{4}, w_{2}$ and $w_{1} w_{2}$ (or $w_{4}, w_{3}$ and $w_{1} w_{3}$ ) would lead to a sixword loop, again a contradiction. If $w_{4}$ contains three of $A, \ldots, F$ (or three of $A, B, C, D, G$ and $H$ ) for which $d$ is of resolution IV, $w_{4}, w_{1}$ and $w_{2}$ (or $w_{4}, w_{1}$ and $w_{3}$ ) would lead to a seven-word group, contradicting the assumption that $G_{4}$ does not contain any seven-word group. In summary, $w_{4}$ contains exactly one letter from each of $A B C D, E F$ and $G H$. Thus $w_{4}$ can be written as $A E G J$ since two letters within each pair $E F$ or $G H$ are symmetric. (It is easy to verify that choosing $w_{4}$ to be $A E G J, B E G J, C E G J$ or DEGJ leads to the same graph.) Then $w_{2} w_{4}$, $w_{3} w_{4}$ and $w_{1} w_{2} w_{3} w_{4}$ are also length- 4 words. Hence $l=9$ and the graph for $G_{4}$ can be represented by Figure 2(k).

Suppose now that $G_{4}$ contains no seven-word group, but it has a six-word loop, which can be written as $A B C D, A B E F, A B G H, C D E F, C D G H$ and $E F G H$. Suppose another independent length-4 word $w$ contains $t$ letters from $A, \ldots, H$. Since $l \geq 8, t \geq 2$. [Otherwise, the graph for $G_{4}$ would be Figure 2(h) and then $l=7$.] If $t \geq 3$, any of $A B, C D, E F$ or $G H$ cannot occur in $w$. Otherwise, say $A B$ and $C$ occur in $w$, then $w A B C D$ would be a length-2 word, contradicting the assumption that $d$ is of resolution IV. Since the pairs $A B, C D, E F$ and $G H$ are symmetric and two letters within each pair are symmetric, we only need to consider $w=A C E G$ or $A C E J$. Then $A B C D, A B E F$ and $w$ would lead to a seven-word group, contradicting the assumption that $G_{4}$ contains no seven-word group. Thus $t=2$. If $w$ contains one of $A B, C D, E F$ and $G H$, say, $A B$, we have Figure 2(m), and the four vertices can be chosen as $A B C D, A B E F, A B G H$ and $A B J K$. If $w$ contains one of the other 24 2FIs consisting of $A, \ldots, H$, say, $A C$, we have Figure 2(i).

Finally, suppose that $G_{4}$ contains a seven-word group, which can be written as $A B C D, A B E F, A C E G, C D E F, B D E G, B C F G$ and $A D F G$. Since all two-letter 
combinations of $A, \ldots, G$ occur twice in the group, if another length-4 word $w$ contains, for example, $A B, w$ cannot contain any of $C, \ldots, F$. Thus, $w$ can have only $t \leq 3$ letters from $A, \ldots, G$. If $t=0$ or 1 , we have Figure 2(j). If $t=2$, we have Figure 2(1), with four vertices $A B C D, A B E F, A C E G$ and $A B H J$. If $t=3$, we have Figure 2(n), with vertices $A B C D, A B E F, A C E G$ and $B C E H$.

From the above graphs, it is easy to obtain the number of unclear 2FIs, $U(d)$, when $G_{m}=L_{4}$ and $d$ has $m \leq 4$ independent length- 4 words. In this case, $l=A_{4}(d)$. If a graph does not contain any three-vertex loop (a six-word loop or a seven-word group) or unfilled boxes, $U(d)=6 m+3(l-m)=3(m+l)$, where $m$ is the number of vertices and $l-m$ is the number of lines. This includes Figures 1(a)-(f), 2(a) and 2(c)-(g). If a graph contains only one three-filledvertex loop (a six-word loop), $U(d)=6 m+3(l-m)+1=3(m+l)+1$. This includes Figures $1(\mathrm{~g}), 2(\mathrm{~h})$ and 2(i), and correspondingly, $U(d)=28,34$ and 37. Figures 1(h), 2(n), 2(k) and 2(m) contain 7, 8, 9 and 10 letters, respectively. Since any 2FIs involving these letters are unclear, then correspondingly, $U(d)=$ 21, 28, 36 and 45. Finally, by the above descriptions of Figures 2(b), (j) and (l), we have $U(d)=30,27$ and 34 , respectively.

3. MA and MaxC2 designs. Based on the graphs constructed in the last section, the following propositions are established. They identify some cases in which an MA $2^{k-p}$ design denoted as $d_{\mathrm{MA}}=d_{\mathrm{MA}}(k, p)$ is also a MaxC2 design.

PROPOSITION 3.1. If $A_{4}\left(d_{\mathrm{MA}}\right)=1$ or $2, d_{\mathrm{MA}}$ is a MaxC2 design and its number of clear 2 FIs is $k(k-1) / 2-6 A_{4}\left(d_{\mathrm{MA}}\right)$.

PROOF. Clearly the proposition holds for $A_{4}\left(d_{\mathrm{MA}}\right)=1$. For $A_{4}\left(d_{\mathrm{MA}}\right)=2$, $d_{\mathrm{MA}}$ has 12 unclear 2 FIs. For any $2_{\mathrm{IV}}^{k-p} \operatorname{design} d, A_{4}(d) \geq A_{4}\left(d_{\mathrm{MA}}\right)=2$. Thus either Figure 1(b) or 1(c) is a subgraph of $d$, which has at least 12 unclear 2FIs. This proves the proposition.

Proposition 3.2. Let $d$ be a $2_{\mathrm{IV}}^{k-p}$ design. If $A_{4}(d) \geq 3, d$ has at least 15 unclear 2 FIs. If $A_{4}(d) \geq 4, d$ has at least 21 unclear $2 F I s$.

PROOF. For $A_{4}(d) \geq 3$, either Figure 1(c) or 1(d) is a subgraph of $d$, which has 15 or 18 unclear 2FIs, respectively. For $A_{4}(d) \geq 4$, if any two length- 4 words do not share two common letters, Figure 2(a) or 2(b) is a subgraph of $d$ and hence $U(d) \geq 24$. Otherwise, there exist three independent length-4 words with at least two of them sharing two common letters. In this case, one of Figures 1(e)-(h) is a subgraph of $d$, and correspondingly, $U(d) \geq 21,24,28$ or 21 .

COROLlary 3.1. For $A_{4}\left(d_{\mathrm{MA}}\right)=3$ or 4 , if there exist two length-4 words with two common letters, $d_{\mathrm{MA}}$ is a MaxC2 design and its number of clear 2FIs is $k(k-1) / 2-6 A_{4}\left(d_{\mathrm{MA}}\right)+3$. 
PROOF. In this case, the graph of $d_{\mathrm{MA}}$ is Figure 1(c) or 1(e), and thus $U\left(d_{\mathrm{MA}}\right)=15$ or 21 , respectively. The corollary then follows from Proposition 3.2.

PROPOSITION 3.3. If $A_{4}\left(d_{\mathrm{MA}}\right)=3$ and $A_{4}\left(d_{\mathrm{MA}}(k-1, p-1)\right)=2, d_{\mathrm{MA}}$ is a MaxC2 design and its number of clear 2 FIs is $k(k-1) / 2-18$.

Proof. It suffices to show that $U(d) \geq 18$ for any $22_{\mathrm{IV}}^{k-p}$ design $d$. Note that $A_{4}(d) \geq A_{4}\left(d_{\mathrm{MA}}\right)=3$. If $A_{4}(d) \geq 4$, by Proposition 3.2, $U(d) \geq 21$. If $A_{4}(d)=3$, it suffices to show that any two length-4 words of $d$ do not share any common letter, say $A$. Otherwise, all the words of $d$ that do not contain letter $A$ would form the defining contrast subgroup of a $2_{\mathrm{IV}}^{(k-1)-(p-1)}$ design $d_{1}$ with $A_{4}\left(d_{1}\right) \leq 1$. This would imply that $A_{4}\left(d_{\mathrm{MA}}(k-1, p-1)\right) \leq 1$, which contradicts the assumption. Thus, each of the three length-4 words of $d$ has 6 unclear 2FIs and none of them overlap, implying that $U(d)=18$.

Recall that, for $k_{\max }(k-p)<k \leq 2^{k-p-2}+1$, there exist resolution IV designs with clear 2FIs. So far, MA designs with $k_{\max }(k-p)<k \leq 2^{k-p-2}+1$ have been obtained only for 15 cases: 32 - and 64-run designs, and 128-run designs with $k=12,13$ and 14. These are given in Tables 4A.3, 4A.5 and 4A.7 of Wu and Hamada (2000). It follows from the above results that the following MA designs are also MaxC2 designs: $2^{7-2}, 2^{9-3}, 2^{10-4}, 2^{12-5}, 2^{13-6}$ (by Proposition 3.1); $2^{8-3}, 2^{11-5}$ (by Corollary 3.1); and $2^{14-7}$ (by Proposition 3.3). Proposition 3.4 below shows that the MA $2^{12-6}$ design is also a MaxC2 design. None of the other six MA designs (for $2^{9-4}, 2^{13-7}, 2^{14-8}, 2^{15-9}, 2^{16-10}$ and $2^{17-11}$ ) are MaxC2 designs. First we prove the following lemmas.

LEMMA 3.1. (i) For any $2_{\mathrm{IV}}^{12-6}$ design d, if a letter, say A, occurs in a length-4 word, there are at least four length-4 words that do not contain letter A.

(ii) For any $2_{\mathrm{IV}}^{12-6}$ design $d$, we have $A_{4}(d) \geq 6$.

Proof. (i) All the words of $d$ that do not contain letter $A$ form the defining contrast subgroup of a $2_{\mathrm{IV}}^{11-5}$ design $d_{1}$. Since $A_{4}\left(d_{1}\right) \geq A_{4}\left(d_{\mathrm{MA}}(11,5)\right)=4$ [see Chen (1992)], $d_{1}$ has at least four length-4 words, which are also the length-4 words of $d$ that do not contain letter $A$.

(ii) By part (i), $A_{4}(d) \geq 5$. Since $d$ has 12 factors (letters), then at least two length- 4 words share a common letter, say $A$. By part (i), there are at least four length-4 words without letter $A$. Hence $A_{4}(d) \geq 6$.

LEMMA 3.2. Figure 2(j) cannot be the graph of any $2_{\mathrm{IV}}^{12-6}$ design $d$.

PROOF. Suppose otherwise that the graph of a $2_{\mathrm{IV}}^{12-6}$ design $d$ is Figure 2(j), which has three connected vertices $w_{1}=A B C D, w_{2}=A B E F$ and $w_{3}=A C E G$. 
Then $w_{4}=w_{1} w_{2}=C D E F, w_{5}=w_{1} w_{3}=B D E G, w_{6}=w_{2} w_{3}=B C F G$ and $w_{7}=$ $w_{1} w_{2} w_{3}=A D F G$. If the isolated vertex $w_{8}$ contains any letter from $A, \ldots, G$, say, $A$, only three length- 4 words $\left(w_{4}, w_{5}\right.$ and $\left.w_{6}\right)$ do not contain letter $A$, contradicting Lemma 3.1(i). Thus $w_{8}$ must contain four letters from $H, J, K$, $L, M$, say $w_{8}=H J K L$. Since $p=6$, there are two other independent words, $w_{9}$ and $w_{10}$, at least one of which (say $w_{9}$ ) does not contain letter $M$. (Otherwise, we can replace $w_{9}$ by $w_{9} w_{10}$.) Furthermore, we can assume $w_{9}$ contains at most two letters from $H, J, K, L$. (Otherwise, we can replace $w_{9}$ by $w_{8} w_{9}$.)

Let $S_{1}=\left\{w_{1}, \ldots, w_{7}\right\}, S_{2}=\left\{w_{9}, w_{9} w_{1}, \ldots, w_{9} w_{7}\right\}$ and $S=S_{1} \cup S_{2}$. Note that $A, \ldots, G$ occur $7 \times 8=56$ times in $S$ and $7 \times 4=28$ times in $S_{1}$. Thus they occur $56-28=28$ times in $S_{2}$. Then there exists $w_{*} \in S_{2}$ that contains at most three letters from $A, \ldots, G$. (Otherwise, $A, \ldots, G$ would occur at least $8 \times 4=32$ times in $S_{2}$.) Since all words in $S_{2}$ contain at most two letters from $H, J, K, L$ and $w_{*}$ has length at least $5, w_{*}$ must contain three letters from $A, \ldots, G$ and two letters from $H, J, K, L$. If $w_{*}$ contains any of the 28 threeletter combinations of $A, \ldots, G$ other than $A B G, A C F, B C E, A D E, B D F, C D G$ and $E F G$, multiplying $w_{*}$ with one of $w_{1}, \ldots, w_{7}$ reduces the length of $w_{*}$ by 2, leading to a length-3 word. This contradicts the assumption that $d$ is of resolution IV. Thus $w_{*}$ can be written as $A B G H J$. Then $S_{3}=\left\{w_{*}, w_{1} w_{*}, \ldots, w_{7} w_{*}\right\} \backslash$ $\left\{w_{4} w_{*}\right\}=\{A B G H J, C D G H J, E F G H J, B C E H J, A D E H J, A C F H J, B D F H J\}$.

Consider another independent word $w_{10}$ with $t$ letters from $A, \ldots, G$. Note that all 21 two-letter combinations of $A, \ldots, G$ occur in $S_{3}$. If $t \geq 2$, multiplying $w_{10}$ with one of the words in $S_{3}$ gives a word $w_{10}^{*}$ which reduces $t$ by at least 1 . Thus we only need to consider $t \leq 1$. Furthermore, $w_{10}$ contains at most three letters from $H, J, K, L, M$. (Otherwise, we can replace $w_{10}$ by $w_{8} w_{10}$.) Then $w_{10}$ is of length at most 4, which contradicts the assumptions that $d$ is of resolution IV and there are only four independent length- 4 words $\left(w_{1}, w_{2}, w_{3}\right.$ and $\left.w_{8}\right)$. Thus Figure 2(j) cannot be the graph of any $2_{\mathrm{IV}}^{12-6}$ design $d$.

Proposition 3.4. The MA $2^{12-6}$ design $d_{\mathrm{MA}}=d_{\mathrm{MA}}(12,6)$ [see page 197 in Wu and Hamada (2000)] is a MaxC2 design and its number of clear 2FIs is 36.

ProOF. First we show that any $2_{\mathrm{IV}}^{12-6}$ design $d$ has at least four independent defining words of length 4 . Otherwise, since $A_{4}(d) \geq 6$ by Lemma 3.1(ii), the graph of $d$ is Figure 1(g) or 1(h). Let $A B C D$ be a length-4 word of $d$. Then from the description of these two graphs in Section 2, it can be shown that only three length-4 words of $d$ do not contain letter $A$. This contradicts Lemma 3.1(i).

Noting that $U(d)=66-C(d)$, we need to prove $U(d) \geq 30$ for any $22_{\mathrm{IV}}^{12-6}$ design $d$. Suppose that $L_{4}$ contains $s$ different tri-word groups. If $s=0, U(d)=$ $6 A_{4}(d) \geq 6 \times 6=36$. If $s=1, U(d)=6 A_{4}(d)-3 \geq 6 \times 6-3=33$. If $s \geq 2$, one of Figures 2(d)-(n) is a subgraph of $d$. If one of Figures 2(d)-(i) and 2(k)-(m) is a subgraph of $d$, by the discussion in the last paragraph of Section $2, U(d) \geq 30$. 
If Figure 2(n) is a subgraph of $d$, the four vertices can be denoted by $A B C D$, $A B E F, A C E G$ and $B C E H$. Denote the 12 factors by $A, \ldots, H, J, K, L, M$. Since $p=6$, there are two other independent defining words, $w_{9}$ and $w_{10}$, one of which (say $w_{9}$ ) contains at most two letters from $J, K, L, M$. (Otherwise, we can replace $w_{9}$ by $w_{9} w_{10}$.) Since all 56 three-letter combinations of $A, \ldots, H$ occur in the 14 length- 4 words of the subgraph, a product of $w_{9}$ and some of these words can always lead to a word $w^{\prime}$ with at most two letters from $A, \ldots, H$. Then $w^{\prime}$ is a length-4 word with two letters from $J, K, L, M$, say $w^{\prime}=A B J K$ (since $d$ is a resolution IV design). This leads to $J K=A B=C D=E F=G H$, which implies that any 2FIs consisting of $A, \ldots, H, J, K$ are unclear. Thus $U(d) \geq 45$.

It remains to consider Figure 2(j) being a subgraph of $d$, which has a seven-word group $w_{1}, \ldots w_{7}$, as written in the proof of Lemma 3.2. This gives 21 unclear 2FIs consisting of $A, \ldots, G$. By Lemma 3.2, $d$ has two other length-4 words, $v_{1}$ and $v_{2}$ (in addition to $w_{1}, \ldots, w_{7}$ ). If $v_{1}$ or $v_{2}$ shares two common letters with any of $w_{1}, \ldots, w_{7}$, since Figure $2(\mathrm{j})$ is a subgraph of $d$, the graph of $d$ contains Figure 2(l) or 2(n) and a separate vertex, whose $U(d) \geq 34+6=40$ or $28+6=34$ as shown in Section 2. Otherwise, the 21 2FIs and the 12 2FIs for $v_{1}$ and $v_{2}$ (or 15 2FIs for $v_{1}, v_{2}$ and $v_{1} v_{2}$ if $v_{1} v_{2}$ is a length-4 word) do not overlap, and thus $U(d) \geq 21+12=33$.

4. MaxC2 designs. The graph representation and classification of length-4 words developed in Section 2 allows us to obtain bounds for the number of clear 2FIs and to reduce the search for designs to a much smaller set. The reduction is usually substantial enough to identify a large MaxC2 design with a complete computer search over the much smaller set. An example is the search for and proof of a MaxC2 $2^{15-8}$ design given below. In this section, we will also develop some useful combinatorial identities and inequalities and group-theoretic arguments to reduce the number of candidate designs.

LEMMA 4.1. (i) For any $2_{\mathrm{IV}}^{14-7}$ design $d_{1}, A_{4}\left(d_{1}\right) \geq 3$. If $A_{4}\left(d_{1}\right)=3$, any two of the three length-4 words of $d_{1}$ cannot share a common letter.

(ii) For any $2_{\mathrm{IV}}^{15-8}$ design $d$, we have $A_{4}(d) \geq 5$.

(iii) Any $2_{\mathrm{IV}}^{15-8}$ design $d$ has at least four independent defining words of length-4.

Part (i) follows from Chen [(1998), page 1269] and is used to prove parts (ii) and (iii). Their proofs are based on the same methods for proving Lemma 3.1 and the first statement in the proof of Proposition 3.4 and are thus omitted.

LEMMA 4.2. Figure 2(c) cannot be the graph of any $2_{\mathrm{IV}}^{15-8}$ design $d$.

Proof. For design $d$, let $1, \ldots, 7$ denote the seven independent columns and let $A, \ldots, H$ denote the other eight columns. Assume that Figure 2(c) is the graph of $d$. Then $A_{4}(d)=5$ and the tri-word group in the graph can be written as $123 A$, 
$124 B$ and $34 A B$. Let $w_{1}$ and $w_{2}$ denote the other two length- 4 words. All the words of $d$ that do not contain letter $A$ form the defining contrast subgroup of a $2_{\text {IV }}^{14-7}$ design $d_{1}$, which contains three length- 4 words: $124 B, w_{1}$ and $w_{2}$. By Lemma 4.1(i), $w_{1}$ and $w_{2}$ do not share a common letter and neither of them contains any of $1,2,4, B$. Similarly, we can show that none of $1,2,3, A$ occurs in $w_{1}$ or $w_{2}$. Thus, the five length- 4 words of $d$ can be written as $123 A, 124 B$, $34 A B, 567 C$ and $E F G H$. Thus $A=123, B=124, C=567$ and $H=E F G$, and we only need to look for the other 4 columns $D, E, F$ and $G$. The only feasible columns that can be used to define $D, E, F$ or $G$ are those consisting of (1) two of $1,2,3,4$ and two of 5, 6, 7; (2) one of 134 and 234 and at least one of 5, 6, 7 and (3) 1234 and two of 5,6,7. We thus end up with choosing 4 out of only 35 columns. This makes a complete computer search an easy task. A program written for this purpose verified that none of the choices can lead to a resolution IV design with the graph of Figure 2(c).

LEMMA 4.3. Figure 2(j) cannot be the graph of any $2_{\mathrm{IV}}^{15-8}$ design $d$.

The proof of Lemma 4.3 is similar to that of Lemma 4.2 (and also with the help of a computer search) and is thus omitted.

Proposition 4.1. The $22_{\mathrm{IV}}^{15-8}$ design with $A=123, B=124, C=134$, $D=234, E=1256, F=1357, G=1467$ and $H=1234567$ is a MaxC2 design with 77 clear 2 FIs.

Proof. For any 2 IV ${ }^{15}$ design $d$, by Lemma $4.1, A_{4}(d) \geq 5$ and $d$ has at least four independent length-4 words. By Lemmas 4.2 and 4.3, neither Figure 2(c) or 2(j) can be the graph of $d$. Thus one of Figures 2(b), 2(d)-2(i) and 2(k)-2(n) is a subgraph of $d$. By the discussion in the last paragraph of Section $2, U(d) \geq 28$. Note that $U(d)=28$ if and only if Figure 2(n) is the graph of $d$. The design given in the proposition is a $2_{\mathrm{IV}}^{15-8}$ design with 28 unclear 2 FIs and is thus a MaxC2 design. [It is obtained by first assuming that Figure 2(n) is the graph of a 2 IV design and going through the same process as for Figures 2(c) and 2(j). The design given above is then obtained.]

We now develop some useful combinatorial identities and inequalities. These results, together with some group-theoretic arguments, are used to show that a class of designs due to Tang, Ma, Ingram and Wang (2002) and the second $2^{9-4}, 2^{13-7}$, $2^{16-10}$ and $2^{17-11}$ designs given in Wu and Hamada [(2000), pages 195 and 197] are MaxC2 designs. Some notation from Cheng, Steinberg and Sun (1999) will be used. For a $2_{\mathrm{IV}}^{k-p}$ design $d$, let $f=2^{k-p}-1-k$ and $m_{j}(d)$ be the number of 2FIs in the $j$ th alias set not containing main effects, where $j=1, \ldots, f$. Furthermore, we define $N_{i}=\#\left\{1 \leq j \leq f: m_{j}(d)=i\right\}$ for $i \geq 0$. Then $N_{i}(i \geq 1)$ is the number of alias sets that contain $i$ 2FIs and $N_{1}=C(d)$ is the number of clear 2FIs. Let $I(d)$ denote the number of 2FIs of $d$. Then $U(d)=I(d)-C(d)$. 
Note that any 2FIs in the same alias set do not share a common letter. Thus $m_{j}(d) \leq k / 2$ and $N_{i}=0$ for $i>r$, where $r$ is the integral part of $k / 2$. Then

$$
I(d)=k(k-1) / 2=\sum_{i=1}^{r} i N_{i}=C(d)+\sum_{i=2}^{r} i N_{i}
$$

and

$$
f=2^{k-p}-k-1=\sum_{i=0}^{r} N_{i}=N_{0}+C(d)+\sum_{i=2}^{r} N_{i} .
$$

It follows from Cheng, Steinberg and Sun (1999) that

$$
A_{4}(d)=\frac{1}{6}\left[\sum_{j=1}^{f} m_{j}(d)\left\{m_{j}(d)-1\right\}\right]=\frac{1}{6}\left\{\sum_{i=2}^{r} i(i-1) N_{i}\right\} .
$$

We have the following lemmas.

LEMMA 4.4. If $N_{i}=0$ for $i>4, N_{2}$ is a multiple of 3 .

Proof. If $N_{i}=0$ for $i>4$, it follows from (4.3) that

$$
A_{4}(d)=\frac{1}{6}\left(2 N_{2}+6 N_{3}+12 N_{4}\right)=\frac{1}{3} N_{2}+N_{3}+2 N_{4} .
$$

Thus $N_{2}=3\left\{A_{4}(d)-N_{3}-2 N_{4}\right\}$, implying that $N_{2}$ is a multiple of 3 .

LEMMA 4.5. If $N_{i}>0$ for some $i$, where $2 \leq i \leq r, U(d) \geq i(2 i-1)$.

Proof. If $N_{i}>0$, there exists an alias set with $i$ 2FIs. These 2FIs contain $2 i$ letters, any two of which form an unclear 2FI. Thus $U(d) \geq\left(\begin{array}{c}2 i \\ 2\end{array}\right)=i(2 i-1)$.

LEMMA 4.6. (i) If $N_{i}=0$ for $i=j+1, \ldots, r$, where $2<j<r$, then

$$
C(d) \leq\left\{(j-1) f+N_{j}-I(d)\right\} /(j-2) .
$$

(ii) If $N_{i}=0$ for $i=j, \ldots, r$, where $2<j \leq r$, then

$$
C(d) \leq\{(j-1) f-I(d)\} /(j-2) .
$$

Proof. It follows from (4.1) and (4.2) that

$$
\begin{aligned}
I(d) & =C(d)+j N_{j}+\sum_{i=2}^{j-1} i N_{i} \\
& \leq C(d)+j N_{j}+(j-1) \sum_{i=2}^{j-1} N_{i} \\
& =C(d)+j N_{j}+(j-1)\left\{f-N_{0}-C(d)-N_{j}\right\} \\
& =C(d)+N_{j}+(j-1)\left\{f-N_{0}-C(d)\right\} \\
& \leq C(d)+N_{j}+(j-1)\{f-C(d)\} .
\end{aligned}
$$


Thus, $C(d) \leq\left\{(j-1) f+N_{j}-I(d)\right\} /(j-2)$. This proves part (i). Part (ii) follows from part (i) by noting that $N_{j}=0$.

Proposition 4.2. For any $2_{\mathrm{IV}}^{k-p}$ design $d$ with $k=2^{k-p-2}+1$, we have $C(d) \leq 2 k-3$. If $C(d)=2 k-3, N_{r-1}=k-2$ and $N_{i}=0$ for $i \neq 1, r-1$.

PROOF. Note that $r=(k-1) / 2>2$ and $f=2^{k-p}-1-k=3 k-5$. If $N_{r}>0$, by Lemma 4.5, $U(d) \geq r(2 r-1)=(k-1)(k-2) / 2$. Thus $C(d)=$ $I(d)-U(d)=k(k-1) / 2-U(d) \leq k-1$. If $N_{r}=0$, by Lemma 4.6(ii),

$$
\begin{aligned}
C(d) & \leq \frac{(r-1) f-I(d)}{r-2}=\frac{\{(k-1) / 2-1\}(3 k-5)-k(k-1) / 2}{(k-1) / 2-2} \\
& =2 k-3>k-1 .
\end{aligned}
$$

That is, $C(d) \leq 2 k-3$. If $C(d)=2 k-3, C(d)=\{(r-1) f-I(d)\} /(r-2)$, and hence $I(d)=C(d)+(r-1)\{f-C(d)\}$. Together with the inequalities in (4.4) (with $j=r$ ), this implies that $N_{r-1}=k-2$ and $N_{i}=0$ for $i \neq 1, r-1$.

COROllary 4.1. The second $2_{\mathrm{IV}}^{9-4}$ and $2 \mathrm{IV}^{17-11}$ designs given in $\mathrm{Wu}$ and Hamada [(2000), pages 195 and 197] are MaxC2 designs.

Proof. These two designs have 15 and 31 clear 2FIs (see the tables there), respectively. The corollary then follows from Proposition 4.2, which implies that, for any $2_{\mathrm{IV}}^{9-4}$ design $d_{1}$ and $2_{\mathrm{IV}}^{17-11}$ design $d_{2}, C\left(d_{1}\right) \leq 2 \times 9-3=15$ and $C\left(d_{2}\right) \leq 2 \times 17-3=31$.

COROLlary 4.2. The $2_{\mathrm{IV}}^{k-p}$ designs $d$ with $k=2^{k-p-2}+1$ given by Tang, Ma, Ingram and Wang (2002) are MaxC2 designs.

PROOF. It follows from Proposition 4.2 by noting that these designs have $C(d)=2 k-3$ as shown by their authors.

Proposition 4.3. The second $2_{\mathrm{IV}}^{13-7}$ design given in Wu and Hamada [(2000), page 197] is a MaxC2 design with 36 clear 2FIs.

To save space, the proof of Proposition 4.3 is omitted here. A detailed proof is given in $\mathrm{Wu}$ and $\mathrm{Wu}(2000)$.

LEMMA 4.7. For any $2_{\mathrm{IV}}^{k-p}$ design $d$, if there exist two alias sets with six 2 FIs containing the same 12 letters, there are exactly three alias sets with six 2 FIs with these 12 letters. 
Proof. Denote an alias set with six 2FIs by $A B=C D=E F=G H=J K=$ $L M$. It gives 30 additional pairs of aliased 2FIs, that is, $A C=B D, A D=B C, \ldots$, $J L=K M, J M=K L$. Another alias set with six 2FIs containing these 12 letters must consist of three of these pairs, say, $A C=B D=E G=F H=J L=K M$. Then there are seven independent length-4 defining words ( $A B C D, A B E F, A B G H$, $A B J K, A B L M, A C E G$ and $A C J L)$ with the 12 letters, which represent 12 columns of $d$. Since $d$ is of resolution IV, four independent columns (1,2,3 and 4) can define at most four other columns $(123,124,134$ and 234), which gives at most eight columns of $d$. Thus, at least five of the 12 letters correspond to independent columns of $d$, implying that there are at most seven independent length-4 defining words with the 12 letters. Hence there is no other independent length-4 word with four of the 12 letters. In this case, it can be verified (by writing out all alias patterns generated by the seven length-4 defining words) that there are exactly three alias sets with six 2FIs containing the 12 letters.

Proposition 4.4. The second $2 \mathrm{IV}^{16-10}$ design given in Wu and Hamada [(2000), page 197] is a MaxC2 design with 29 clear 2FIs.

Proof. Consider any $2_{\mathrm{IV}}^{16-10}$ design $d$. Then $f=47, I(d)=120$ and $r=8$. It suffices to show that $C(d) \leq 29$ or $U(d) \geq 91$. If $N_{8}>0$, by Lemma 4.5, $U(d) \geq 120$. If $N_{8}=0$ and $N_{7}>0$, again by Lemma 4.5, $U(d) \geq 91$. If $N_{8}=$ $N_{7}=0$, by Lemma 4.6(i), $C(d) \leq\left\{5 f+N_{6}-I(d)\right\} / 4=\left(5 \times 47+N_{6}-120\right) / 4=$ $29.75+\left(N_{6}-4\right) / 4$. Thus $C(d) \leq 29$ for $N_{6} \leq 4$.

It remains to consider the case in which $N_{8}=N_{7}=0$ and $N_{6} \geq 5$. Denote an alias set with six 2FIs by $A B=C D=E F=G H=J K=L M$. Since $N_{6} \geq 5$, by Lemma 4.7, there exists another alias set with six 2FIs and $t \geq 2$ new letters (other than $A, \ldots, H, J, K, L, M$ ). (It cannot contain only one new letter since the 60 additional aliased 2FIs, $A C=B D, A D=B C, \ldots, J L=K M, J M=K L$, occur pairwise in an alias set.) If $t \geq 3, U(d) \geq 66+t(t-1) / 2+t(12-t) \geq 96$. Otherwise, without loss of generality, we have $A C=B D=E G=F H=J P=L Q$. (Choosing $J P=K Q$ gives $A B=C D=E F=G H=J K=L M=P Q$, contradicting the assumption that $N_{8}=N_{7}=0$.) Then $K M=J L=P Q$ and the 912 FIs consisting of $A, \ldots, H, J, K, L, M, P, Q$ are unclear. Thus $U(d) \geq 91$, proving the proposition.

5. Summary and concluding remarks. Proving whether a design is a MaxC2 design is technically challenging. Unlike the MA criterion which is defined in terms of the word-length pattern, the number of clear 2FIs is a complicated mathematical function of the defining contrast subgroup. This technical difficulty may explain why there has been no general method for tackling the problem. In this paper, we give a method for proving whether a resolution IV design is a MaxC2 design. First we develop a graph representation and classification of length-4 words. This representation of designs allows us to obtain bounds for the 
number of clear 2FIs and to reduce the search for designs to a much smaller set. We also develop some useful combinatorial identities and inequalities (as given in Section 4) and group-theoretic arguments (as used in Sections 3 and 4) to reduce the number of candidate designs. If theoretical arguments alone cannot do the job, the reduction is usually substantial enough for computer search to finish the job. An example is the search for and proof of a MaxC2 $2^{15-8}$ design in Section 4.

In addition to proving the MaxC2 property of many existing designs, we are able to find new MaxC2 designs such as a class of designs due to Tang, Ma, Ingram and Wang (2002) and the $2^{15-8}$ design mentioned above. It demonstrates that the tools can be effectively used to find new MaxC2 designs. We have given lengthy proofs for several designs (known to be MaxC2 designs by computer search) in order to show how the approach can be implemented. Only through the demonstration in these proofs will the potential users be able to use and improve the tools to find designs for new situations such as 128- and 256-run designs, three-level designs, two-level designs in blocks or with control and noise factors for parameter design application. It is our hope that this work is a modest start of a new direction of research in fractional factorial designs.

Acknowledgments. Thanks to the referees for their constructive comments and suggestions, and to D. X. Sun for computing assistance.

\section{REFERENCES}

Bose, R. C. (1947). Mathematical theory of the symmetrical factorial design. Sankhyā 8 107-166.

Chen, H. and Hedayat, A. S. (1998). $2^{n-m}$ designs with resolution III or IV containing clear two-factor interactions. J. Statist. Plann. Inference 75 147-158.

CHEN, J. (1992). Some results on $2^{n-k}$ fractional factorial designs and search for minimum aberration designs. Ann. Statist. 20 2124-2141.

CHEN, J. (1998). Intelligent search for $2^{13-6}$ and $2^{14-7}$ minimum aberration designs. Statist. Sinica 8 1265-1270.

Chen, J., Sun, D. X. and Wu, C. F. J. (1993). A catalogue of two-level and three-level fractional factorial designs with small runs. Internat. Statist. Rev. 61 131-145.

Cheng, C.-S., Steinberg, D. M. and Sun, D. X. (1999). Minimum aberration and model robustness for two-level fractional factorial designs. J. Roy. Statist. Soc. Ser. B 61 85-93.

FrIes, A. and Hunter, W. G. (1980). Minimum aberration $2^{k-p}$ designs. Technometrics $22601-$ 608.

TANG, B., MA, F., INGRAM, D. and WANG, H. (2002). Bounds on the maximum number of clear two-factor interactions for $2^{m-p}$ designs of resolution III and IV. Canad. J. Statist. 30 $127-136$.

Wu, C. F. J. and Hamada, M. (2000). Experiments: Planning, Analysis, and Parameter Design Optimization. Wiley, New York.

WU, H. and WU, C. F. J. (2000). Clear two-factor interactions and minimum aberration. Technical Report 363, Dept. Statistics, Univ. Michigan, Ann Arbor.

DEPARTMENT OF STATISTICS

IOWA STATE UNIVERSITY

AMES, IOWA 50011-1210

E-MAIL: isuhwu@iastate.edu
DEPARTMENT OF STATISTICS

UNIVERSITY OF MICHIGAN

ANN ARbor, Michigan 48109-1285

E-MAIL: jeffwu@umich.edu 